\title{
Modernités multiples et mondialisation
}

Multiple Modernities and Globalization

\section{Matthias Koenig}

\section{OpenEdition}

Journals

Édition électronique

URL : http://journals.openedition.org/assr/26158

DOI : $10.4000 /$ assr. 26158

ISSN : 1777-5825

Éditeur

Éditions de l'EHESS

\section{Édition imprimée}

Date de publication : 20 octobre 2014

Pagination : 127-145

ISBN : 978-2-7132-2433-1

ISSN : 0335-5985

\section{Référence électronique}

Matthias Koenig, « Modernités multiples et mondialisation », Archives de sciences sociales des religions [En ligne], 167 | juillet-septembre 2014, mis en ligne le 20 octobre 2017, consulté le 02 mai 2019. URL: http://journals.openedition.org/assr/26158; DOI : 10.4000/assr.26158 


\section{Matthias Koenig}

\section{Modernités multiples et mondialisation ${ }^{1}$}

L'horizon d'attente dans lequel se situent les débats allemands en sociologie des religions est marqué, comme ailleurs, par une perte de plausibilité des autodéfinitions courantes du monde occidental moderne et des catégories de la sociologie classique. D’abord un discours amorcé dès les années 1980 pose un diagnostic sur l'époque, la qualifie de "postmodernité », de modernité seconde ou encore de modernité réflexive. Ce discours révèle la prise de conscience d'une césure avec la modernité européenne, désormais perçue comme hautement contingente. Ensuite, très en lien aussi avec ce discours, l'expérience - liée à la technologie - de la densification de la communication, des échanges et des interdépendances entre sociétés a été thématisée au cours des années 1990 par la notion de mondialisation, accompagnée par une critique (parfois trop) vive à l'encontre du "nationalisme méthodologique » des sciences sociales. Enfin, les études postcoloniales ont durablement relativisé, notamment hors de l'Occident, la signification supposée universelle des pratiques et des discours sociaux inscrits dans la modernité européenne.

Dans la mesure où ces trois évolutions ont renforcé la critique des théories conventionnelles de la modernisation (S. Kaviraj, 2005 ; W. Knöbl, 2001 et 2007), on ne saurait s'étonner que les cadrages conceptuels en sociologie des religions aient eux aussi été déstabilisés. Comme dans d'autres contextes nationaux, un débat complexe s'est développé en Allemagne autour de la critique de la théorie conventionnelle de la sécularisation (voir notamment D. Pollack, 2003). D’une part, la thèse d'un déclin des pratiques et des croyances a été confrontée à la thèse de l'individualisation (T. Luckmann, 1991) et à la théorie du marché religieux (pour le débat voir D. Pollack, 2009, ainsi que M. Koenig, C. Wolf, 2013). D'autre part, on a interrogé le rôle du religieux dans l'émergence et les transformations structurelles et culturelles de la modernité. Ce dernier aspect a

1. Cet article est une version retravaillée d'un texte allemand publié dans le volume Kulturen der Moderne, dirigé par Thorsten Bonacker et Andreas Reckwitz (Frankfurt am Main, New York : Campus, 2007). Je remercie Anne-Sophie Lamine et les deux lecteurs anonymes des Archives pour leurs excellentes remarques et suggestions, et Marie Gravey pour m'avoir assisté pour la traduction française.

ARCHIVES DE SCIENCES SOCIALES DES RELIGIONS 167 (juillet-septembre 2014), p. 127-145 
suscité un intérêt particulier dans la sociologie allemande, en dehors même de la sociologie des religions, parce qu'il s'inscrit nettement dans la problématique de la rationalisation, héritage des travaux de Max Weber.

Mise à l'épreuve par la réflexion sur la modernité, par la mondialisation et par la critique postcoloniale d'une modernité " universaliste » occidentale, l'approche weberienne a été prolongée de deux manières différentes. Un premier prolongement consiste à appréhender explicitement la modernité contemporaine comme société mondiale, donc à souligner la portée universelle de dynamiques comme la différenciation fonctionnelle ou la rationalisation culturelle. On trouve plusieurs versions d'une telle théorie de la société-monde (weltgesellschaft), dans l'œuvre monumentale de Niklas Luhmann et dans la théorie néo-institutionnaliste de John W. Meyer qui jouissent d'un grand intérêt académique (voir B. Heintz et al., 2005 ; T. Wobbe, 2000). On a commencé à étudier les dynamiques mondiales du religieux en s'intéressant aux circulations des catégories du " religieux ", aux mouvements religieux transnationaux, aux diasporas, etc. Un second prolongement vise à donner une vitalité nouvelle à la comparaison des civilisations, tout en soulignant la divergence culturelle ainsi que structurelle des sociétés modernes. C'est la théorie des « modernités multiples » de Shmuel N. Eisenstadt, qui, en s'appuyant sur des travaux interdisciplinaires autour du comparatisme historique inspiré par Weber (W. Schluchter, 1988), a, peut-être plus qu'ailleurs, influencé les débats théoriques. Le débat récent sur la comparaison des "sécularités» montre bien la pertinence de cette approche (M. Wohlrab-Sahr, M. Burchardt, 2011 ; pour une perspective française voir D. Hervieu-Léger, 2003).

Quelques auteurs ont tenté une synthèse entre ces courants en mettant en dialogue la théorie de la différentiation fonctionnelle selon Niklas Luhmann (1975 et 1997 ; cf. aussi R. Stichweh, 2000) avec l'approche des modernités multiples selon Eisenstadt. Ainsi, dans une démarche de reconstitution critique de la théorie de la différenciation, Thomas Schwinn $(2001 ; 2003$; 2005) affirme qu'une conjugaison des deux approches a l'avantage de faire découler la diversité de la modernité de l'aptitude variable à la mondialisation de différents soussystèmes fonctionnels ${ }^{2}$. C'est cependant avec la théorie néo-institutionnaliste de la société mondiale de John W. Meyer que l'on propose ici de mettre la théorie des «modernités multiples " en perspective. On explicitera d'abord les raisons de se focaliser sur ces deux approches théoriques macrosociologiques qui s'inscrivent toutes les deux, bien que très différemment, dans la tradition weberienne. On montrera ensuite comment une synthèse théorique de ces deux approches est possible du fait de prémisses communes et permet en outre de résoudre des problèmes intrinsèques à ces théories. On esquissera enfin le

2. Au sein même de la théorie des systèmes, se multiplient les signes d'une prise en compte accrue de la diversité de la modernité telle qu'elle a été amenée par Eisenstadt ; voir A. Nassehi, 2003 : 209 sq. 
potentiel d'un programme de recherche s'inspirant de la combinaison de ces théories à partir d'exemples d'analyse historique comparative de variantes institutionnelles et de transformations de la sécularité.

\section{Deux héritages weberiens : néo-institutionnalisme mondial et modernités multiples}

C'est pour deux raisons que l'on choisit ici de combiner l'analyse de civilisation d'Eisenstadt et la théorie de la société mondiale de Meyer pour comprendre les processus divergents et convergents de la rationalisation. La première est d'ordre stratégique et consiste dans le fait que les deux approches ont développé leurs arguments théoriques dans un dialogue étroit avec la recherche empirique. Il est vrai que les deux auteurs ont des préférences méthodologiques divergentes, portant sur les études macro-statistiques d'un côté et sur les analyses historiques comparatives de l'autre. Celles-ci découlent de leurs intérêts scientifiques initiaux pour la convergence ou pour la divergence des structures sociales modernes. Cependant leur orientation résolument empirique rend ces deux approches tout aussi attrayantes en vue de formuler un programme de recherche sur les variantes institutionnelles de la modernité, voire de la sécularité, dans la société mondiale. Elle incite aussi à conjuguer ces deux angles théoriques qui semblent à première vue antinomiques.

La seconde raison est d'ordre systémique. Les deux auteurs portent de manière similaire leur regard sur le cadre culturel ou institutionnel dans lequel s'inscrivent les processus de différenciation fonctionnelle. Ils accordent donc à la "sémantique ", considérée par Luhmann essentiellement comme un dérivé de la "structure sociale », un certain degré d'autonomie causale ${ }^{3}$. Meyer, en s'appuyant sur la genèse du rationalisme occidental reconstituée par Weber, met surtout l'accent sur sa "signification culturelle universelle ». Il estime qu'un système culturel de relations fins-moyens s'est développé, à partir des transformations du christianisme du bas Moyen Âge, d'abord européen puis au centre d'une culture mondiale portée par des organisations internationales, des organisations non-gouvernementales et des professions (J. W. Meyer, 2005). L'institutionnalisation mondiale de modèles culturels cognitifs et normatifs rationalisés a selon lui pour résultat un niveau étonnamment élevé d'isomorphie entre les États, les organisations et même les individus.

Eisenstadt se rattache quant à lui aux études comparatives de Weber et souligne les aspects relativistes de sa théorie de la rationalisation. Il interprète en effet "l'éthique économique des religions mondiales » non pas comme une explication de l'échec de la rationalisation dans les civilisations extra-européennes,

3. Dans la perspective systémique Luhmannienne, chaque système a une "sémantique " propre $[\mathrm{NdE}]$. 
mais comme un point de départ de l'analyse des voies de modernisation et de différenciation divergentes ${ }^{4}$. D'un côté, les religions de la période axiale, avec la tension entre sphères transcendante et séculière, ont en partage une grammaire culturelle commune qui explique la tendance interne au changement et, partant, la réceptivité au projet culturel de la modernité, y compris au-delà de l'« Occident ». D'un autre côté, selon l'auteur, la formulation de cette tension implique des idées différentes sur l'orthodoxie et l'hétérodoxie. Elle légitime également des configurations institutionnelles différentes du marché, du pouvoir et de l'identité collective et établit des intérêts différents des élites et des mouvements de protestation dont l'interaction engendre des modernités multiples (S. Eisenstadt, 2000). Meyer et Eisenstadt se rejoignent ainsi tout en faisant des lectures différentes de la théorie weberienne de la rationalisation. Il reste à savoir si ces deux lectures sont compatibles entre elles.

\section{Cadrages culturels de la modernité entre convergences et divergences}

Pour démontrer qu'une synthèse des approches de Meyer et d'Eisenstadt est possible, il faut commencer par mettre en lumière leurs prémisses théoriques communes. Pour ce faire, il convient d'abord de se demander de quelle manière elles tiennent compte du contexte d'expérience mentionné en introduction. À première vue, on pourrait croire que ces deux approches comptent simplement parmi les nombreux épisodes d'essor et de repli des théories de la modernisation de l'après-guerre. Les premiers écrits d'Eisenstadt publiés dans les années 1950 et 1960 étaient effectivement très marqués par le fonctionnalisme structurel ; de même, les premiers travaux de Meyer avaient des racines évidentes dans la recherche sur l'éducation des années 1970, encore très imprégnée au début d'optimisme et de foi dans le progrès. Mais, à y regarder de plus près, Meyer comme Eisenstadt soumettent l'appareil conceptuel de la sociologie à une révision d'une portée importante et se différencient ainsi du néo-modernisme. Leur démarche s'articule autour d'une théorie réflexive de l'action et des systèmes, d'une définition de la modernité ancrée dans des contextes culturels et d'une focalisation analytique sur la sphère politique.

La distinction opérée par Peter Wagner (2001) entre théories sociales « modernistes » et "modernes» permet d'éclairer l'ancrage fondamental de ces deux approches dans les théories de l'action et des systèmes. D’une part, les théories sociales «modernistes» postulent soit l'autonomie d'un individu agissant de

4. Cet intérêt pour la diversité de la modernité apparaît déjà dans les premières publications d'Eisenstadt ; il se développe au cours des années 1970 (S. Eisenstadt, 1973), mais c'est seulement après l'élaboration de son concept de cultures axiales qu'il connaît une percée essentielle (S. Eisenstadt, 1982, 2003) ; pour une histoire détaillée de son œuvre, cf. M. Koenig, 2005. 
manière rationnelle (utilitarisme), soit la rationalité de l'ordre social (fonctionnalisme, structuralisme) ou bien, dans des variantes "dualistes ", naturalisent des acteurs et des systèmes sociaux rationnels. D'autre part, les théories sociales "modernes » adoptent un plus haut degré de réflexivité. Meyer et Eisenstadt se trouvent clairement dans le second groupe, celui des théories "modernes » ou réflexives. Le néo-institutionnalisme sociologique développé par Meyer conteste résolument l'idée que la sociologie pourrait accepter comme naturellement données les notions d'agency et d'actorhood. Selon Meyer, il convient plutôt d'interpréter les acteurs rationnels comme les produits d'un système culturel historiquement unique de fins légitimes et de relations entre fins et moyens (J. W. Meyer, R. Jepperson, 2000 : 101 ; pour une critique de «l'individualisme méthodologique " voir aussi R. Jepperson, J. W. Meyer, 2011). De même, il faudrait appréhender les théories fonctionnalistes de la société comme une composante de ce cadre culturel de la rationalité, et donc les déplacer vers la "sémantique» (J. W. Meyer, 1999: 126). Eisenstadt historicise lui aussi la confrontation entre action individuelle et système social, caractéristique de la problématisation centrale de la sociologie, et se réfère à la dynamique culturelle des mouvements modernes de protestation (S. Eisenstadt, M. Curelaru, 1976), et même, en dernière instance, à la grammaire culturelle du temps axial, dans laquelle le monde (social) est saisi comme entité nécessitant une reconstruction rationnelle (J. Alexander, 1992 : 88). Meyer et Eisenstadt formulent ainsi l'un comme l'autre, dans la lignée de l'analyse weberienne du rationalisme occidental, une théorie antiévolutionniste de la modernité qui prend en compte la contingence historique des conceptions modernes de l'action et des systèmes rationnels.

En outre, en lien étroit avec cette conscience de la contingence, les deux théoriciens définissent la modernité essentiellement comme une construction culturelle. La théorie de la modernité formulée par Meyer dans le cadre de sa théorie cognitive des institutions peut être comprise comme une interprétation strictement phénoménologique ${ }^{5}$ de la théorie weberienne de la rationalisation. L'auteur considère que la rationalité fonctionne comme un mythe, l'action rationnelle comme son accomplissement rituel ou dramaturgique et la rationalisation, comme l'institutionnalisation d'un système culturel de fins ultimes, de schémas fins-moyens et d'identités rationnelles d'acteurs (J. W. Meyer et al., 1987 ; J. W. Meyer, R. Jepperson, 2000). Eisenstadt interprète lui aussi la modernité comme un projet culturel, mais en insistant sur d'autres aspects. Si Meyer a une approche que l'on peut qualifier de «macro-phénoménologique », Eisenstadt ancre quant à lui sa théorie culturelle, dans le sillage de sa réception de la philosophie sociale de Martin Buber, sur l'idée d'une réflexivité et d'une recherche indispensable du sens de la condition humaine (S. Eisenstadt, 1995 : 1). Le projet culturel de la modernité est selon lui marqué par un ensemble spécifique de prémisses cosmologiques et ontologiques caractérisées par une réflexivité accrue et - on trouve là une référence directe à la sociologie weberienne de la religion -

5. Au sens de la construction sociale de la réalité de Berger et Luckmann (1967). 
par la foi en la réalisation intramondaine de représentations transcendantes. Pour Eisenstadt, c'est l'horizon d'attente ainsi ouvert qui explique la dynamique inédite de transformation inscrite dans une perspective d'histoire universelle et dans le potentiel révolutionnaire des sociétés modernes (S. Eisenstadt, 1978). Malgré les contours différents donnés à leurs théories culturelles, Meyer et Eisenstadt s'accordent à définir la modernité non pas par des configurations institutionnelles spécifiques comme la démocratie, l'économie de marché, le droit formel ou un modèle de différenciation spécifique, mais comme une structure d'interprétation et d'attente qui constitue une diversité de projets de vie et de modelage du monde (weltgestaltung). La culture de la modernité n'est alors ni cohérente ni exempte de contradictions, ainsi que le souligne Eisenstadt (1998) en évoquant les antinomies de la modernité (autonomie versus hétéronomie ; liberté versus égalité ; pluralisme versus jacobinisme), mais aussi Meyer, dans ses analyses matérielles de la culture mondiale (J. W. Meyer et al., 1997 : 170). Cependant, quel qu'en soit le contenu culturel exact, l'aspect décisif est ici que les processus de différenciation fonctionnelle et structurelle soulignés dans les théories conventionnelles de la modernisation naissent précisément de ces modèles de culture spécifiquement modernes.

Ni Meyer ni Eisenstadt n'abusent de la fameuse métaphore weberienne de l'aiguilleur. Ils concentrent leurs analyses empiriques sur les modèles culturels et sur les idées modernes ainsi que sur leurs institutionnalisations. L'accent est surtout mis - et c'est là leur troisième point commun conceptuel - sur la sphère politique comme principal champ de projets de modelage rationnel de la société. Pour Eisenstadt, avec l'entrée dans la modernité, le politique revêt même une qualité charismatique. Il pénètre, comme le montre en particulier la politisation des identités collectives, d'autres pratiques et institutions sociales (S. Eisenstadt, 1998 : 4). De manière similaire, Meyer voit dans la constitution et l'expansion de l'État souverain comme principal acteur collectif de la reconstruction sociale un résultat central de la "dévolution " de l'autorité divine, à l'origine selon lui du rationalisme occidental (G. Thomas, J. W. Meyer, 1984). Du point de vue de Meyer comme d'Eisenstadt, c'est l'État national, combinaison d'un pouvoir politique, d'un système juridique et d'une identité collective, qui constitue le cadre principal du politique. En analysant le développement des États nationaux dans le contexte de civilisations ou de sociétés mondiales, les deux auteurs échappent donc au "nationalisme méthodologique » et aux problèmes émanant d'une naturalisation d'un concept (national) de la société.

Les approches de Meyer et d'Eisenstadt ont donc en commun de souligner la contingence historique, la constitution culturelle et l'institutionnalisation politique de la modernité et, en cela, elles se distinguent clairement des théories conventionnelles de la modernisation. Ces trois points communs confirment l'intuition de départ selon laquelle leur synthèse est pour le moins possible ${ }^{6}$.

6. C'est une vision partagée également par Schwinn, 2005, p. 216. 
Reste à prouver que cette synthèse, en plus d'être possible, est aussi fructueuse et même nécessaire, si l'on souhaite dépasser les points aveugles que comportent chacune des approches. Effectivement, la reconstruction de prémisses théoriques communes ne peut cacher le fait que Meyer et Eisenstadt se concentrent sur des questions analytiques extrêmement différentes. Alors que l'École de Stanford étudie les isomorphies ou convergences résultant de l'institutionnalisation mondiale du rationalisme occidental, Eisenstadt s'intéresse en priorité aux divergences des sociétés modernes, qui reposent sur différentes cultures axiales. On pourrait donc penser qu'on a affaire à des intérêts de recherche non congruents. Cependant, à y regarder de plus près, il apparaît que les deux stratégies explicatives sont non seulement complémentaires, mais même dépendantes l'une de l'autre.

On reproche souvent à cette conception des modernités multiples un manque de cohérence conceptuelle (voir V. Schmidt, 2006 ; B. Wittrock, 2000). Comment l'utilisation du pluriel est-elle compatible avec le fait que l'auteur choisit une définition minimale implicite du projet culturel et politique de la modernité (au singulier) ? Plus important, comment expliquer que la modernité soit devenue un cadre de référence mondial ? Eisenstadt souligne, il est vrai, que la modernité est le premier type de civilisation à revendiquer une portée mondiale, à la différence des religions universelles (S. Eisenstadt, 2003 : 502). Mais il n'étudie généralement pas dans le détail la dynamique de diffusion planétaire du projet moderne. C'est précisément sur ce point que le débat sur les modernités multiples pourrait tirer profit d'une réception plus approfondie de la théorie néoinstitutionnaliste de la société mondiale (world polity). Celle-ci peut par exemple contribuer à préciser les mécanismes de diffusion mondiale du projet culturel de la modernité. Le fait que divers mécanismes - imitation, pression normative et contrainte - articulent les niveaux mondial et local explique comment il est possible, dans des conditions extrêmement hétérogènes, d'avoir une orientation semblable vers la culture moderne. L'analyse néo-institutionnaliste de mécanismes de diffusion permet en outre d'examiner les dynamiques de mondialisation, y compris au sein des différentes civilisations axiales et de suivre l'hypothèse selon laquelle, en réaction à la culture rationnelle occidentale, se constituent des systèmes de référence globaux alternatifs, souvent avec des fondements religieux. Et enfin, l'orientation phénoménologique de la théorie de la société mondiale peut rendre visibles les cadres cognitifs et normatifs généraux sur la base desquels sont constitués, légitimés et naturalisés des unités macrosociales, civilisations inclues. On peut penser ici aussi bien à la sémantique des "religions universelles » de la fin du XIX ${ }^{\mathrm{e}}$ siècle, issue de l'interdépendance mondiale accrue, qu'au discours sur la « lutte » ou le « dialogue » des « civilisations » à la fin du XX $x^{\mathrm{e}}$ siècle.

Réciproquement, une synthèse théorique apparaît aussi nécessaire et fructueuse pour éclairer les points aveugles qui persistent dans l'approche néoinstitutionnaliste de la société mondiale. En particulier, celle-ci n'indique pas comment, au niveau local, les modèles culturels mondiaux font l'objet d'une 
appropriation, d'une interprétation et d'une adaptation. Et c'est précisément en cela que réside un apport possible de la littérature sur les variations de la modernité. Celle-ci souligne en effet la « dépendance au chemin emprunté » (path dependence). L'approche néo-institutionnaliste pourrait profiter de cet apport à trois égards. D'abord, selon la théorie néo-institutionnaliste, les structures organisationnelles formelles sont fortement « dissociées " des activités quotidiennes réelles, dans les champs institutionnels fortement rationalisés (J. W. Meyer et al., 1997 : 160). L'examen plus précis de ce point oblige à identifier clairement les facteurs locaux qui permettent d'expliquer la structure de l'activité réelle et son niveau de résistance plus ou moins élevé face à la structure formelle légitimée par des modèles culturels mondiaux ; ainsi, par exemple, les décalages variables entre le discours globalisé sur les droits des femmes et les réalités constitutionnelles dans divers pays requièrent une attention explicite aux particularités locales (B. Heintz et al., 2006). Les analyses de la "dépendance au chemin emprunté » par les civilisations que rencontrent les modèles culturels mondiaux peuvent apporter ici une connaissance supplémentaire. Les arguments de la path dependence peuvent aussi apporter un éclairage sur les manières d'exploiter stratégiquement à l'échelle locale les contradictions institutionnelles inhérentes à la culture mondiale. Les modèles culturels propres à une civilisation ou une nation comme les constellations d'acteurs et d'intérêts locaux déterminent quels modèles culturels mondiaux peuvent être repris par les élites politiques et par les mouvements de protestation ainsi que la manière dont ils sont interprétés ${ }^{7}$. Enfin, l'autre problème de la théorie néo-institutionnaliste de la société mondiale auquel la prise en compte des dynamiques « civilisationnelles » serait profitable est l'explication de la transformation des schémas, scripts et modèles légitimés dans la culture mondiale. Ainsi, l'intégration des civilisations extra-européennes au système moderne des États dans le sillage de la décolonisation n'a pas été sans altérer la teneur de la culture mondiale; elle a produit de nouvelles structures d'interprétation et d'attente (interdictions de discrimination, droit au développement, etc.) et des conflits correspondants (par exemple R. Burke, 2010).

Si l'on dresse le bilan de l'argumentation conduite jusqu'ici, on constate que des prémisses théoriques communes et des lacunes complémentaires permettent de combiner judicieusement la théorie néo-institutionnaliste de la société mondiale de Meyer et l'analyse comparative des civilisations d'Eisenstadt, et d'en tirer un programme de recherche sur les constructions culturelles et les variantes institutionnelles de la modernité dans la société mondiale - programme qui ouvre de nouvelles pistes de réflexion en sociologie des religions.

7. La littérature néo-institutionnaliste accorde désormais une attention significative aux interactions complexes entre structures mondiales d'attente et d'interprétation (d'une part) et path dependence nationale d'autre part ; cf. par exemple Y. Soysal, 1994. 


\section{La problématique de la sécularisation : constructions culturelles et variations institutionnelles}

Le champ de recherches de la sociologie des religions reste fortement marqué par l'appareil conceptuel moderniste et nécessite de ce fait, comme cela a été suggéré par plusieurs auteurs (par exemple J. Beckford, 2003), une réorientation théorique. Au Xx ${ }^{\mathrm{e}}$ siècle, le paradigme qui a guidé la connaissance était celui de la sécularisation. En s'appuyant sur des théories conventionnelles de la modernisation, on considérait que l'industrialisation, l'urbanisation et la démocratisation conduisaient à la rationalisation, la pluralisation et la privatisation de la religion, voire à sa perte complète de signification, en tout cas à sa différenciation d'avec les autres sphères de valeurs ou sous-systèmes (K. Dobbelaere, 1981 ; D. Pollack, 2003 ; O. Tschannen, 1992). Or, depuis environ deux décennies ce paradigme est remis en question : certains auteurs, Thomas Luckmann étant la référence majeure en Allemagne, contestent la thèse d'un déclin des pratiques et croyances religieuses et proposent d'expliquer comment la modernité est elle-même productrice de nouvelles formes du religieux dans la mesure où elle renforce l'incertitude et la conscience de la contingence. D'autres estiment que des formes publiques de religion, dans lesquelles les prémisses fondamentales de la modernité sont acceptées, sont non seulement empiriquement possibles, mais aussi normativement légitimes - pour autant qu'elles se considèrent comme faisant partie d'une société civile pluraliste et participent du discours rationnel d'une sphère publique autonome (J. Casanova, 1994 : 19-39, 232).

Cependant, malgré ces critiques, le cœur du paradigme moderniste, à savoir la thèse de la différenciation fonctionnelle entre religion et politique, est rarement remis en cause (P. Gorski, 2000). Ainsi, Casanova défend, dans son analyse éclairante de la "dé-privatisation » de la religion, une conception normative de la différenciation qui repose sur une définition moderniste réduisant l'essence de la religion à une sphère de convictions religieuses, de valeurs morales et de pratiques rituelles séparée du pouvoir politique ${ }^{8}$. De même, la définition que propose Hervieu-Léger (1993 : 119, 135) de la religion comme "lignée croyante » sousentend une différenciation du religieux et du politique coïncidant avec la distinction entre hétéronomie et autonomie. Dans les deux cas, le cadre de référence culturel dans lequel des configurations institutionnelles du religieux et du politique sont inscrites n'est pas traité.

L'un des aspects centraux du projet culturel de la modernité et de son institutionnalisation dans l'État territorial et national consiste à imaginer le social comme "séculier» (voir dans ce contexte aussi C. Taylor, 2007). Si l'on suit Eisenstadt, la construction culturelle de la modernité en Europe peut être décrite comme le résultat de mouvements hétérodoxes au sein du christianisme, qui ont tenté de

8. Dans une optique critique, $c f$. T. Asad, 2003, p. 181-201. 
surmonter la tension axiale entre ordres transcendant et immanent par un activisme à la fois radicalisé et intramondain. Les modèles traditionnels de légitimation se sont alors effondrés et les valeurs de la protestation - liberté, égalité, solidarité - sont venues au cœur de l'ordre social. L'entrée dans la modernité transforme fondamentalement les présupposés culturels et le cadre institutionnel de la "religion », comme le montre bien les évolutions sémantiques. En effet, ce n'est que dans le vocabulaire postérieur à la Réforme qu'apparaîssent des traductions en langue vernaculaire du terme latin "religio ", qui prend désormais une signification spécifiquement moderne ${ }^{9}$. Le terme générique désignait des croyances partagées collectivement, faisant l'objet de pratiques rituelles et organisées par une appartenance ( «la religion »), pensées comme dissociées des projets de rationalisation intramondains naissants dans l'économie, la science, la technologie, mais aussi la politique. Dans un second temps, le concept de religion a pris, en particulier dans les contextes de confrontation avec l'Empire ottoman et d'expansion européenne, une signification relativiste ( $u n e$ religion »/ les religions ») permettant de classer différentes modalités de la religion. Étaient alors sousentendus de nouveaux imaginaires du "séculier". Le saeculum, compris dans le récit eschatologique du christianisme latin comme un interlude entre la création et l'eschaton, devient une période qualifiée par la philosophie politique des Lumières puis la sociologie comme la « société " (W. Sewell, 2005 : 325) dans laquelle sont situées et la « religion » et la «politique».

C’est surtout dans le cadre de l'État-nation que la "sécularité » a été institutionnalisée comme « imaginaire » de la modernité. Face à l'Église, la sphère politique a gagné en autonomie. Elle a pris sa place au centre d'une mise en forme rationnelle de la société (gesellschaftgestaltung). Le charisme, qui dans la chrétienté médiévale relevait du pouvoir « religieux » de l'Église (ecclesia), s'est complètement déplacé vers le pouvoir " séculier » (imperium) de l'État ${ }^{10}$. La position de ce qui avait désormais valeur de "religion » devenait tributaire de la séparation politique entre "public » et "privé ». Notons cependant que les communautés de croyants avaient déjà été considérées comme relevant de la sphère privée, au moment des guerres confessionnelles, lorsque l'État absolutiste était censé dépasser leurs caractères particularistes. $\mathrm{Au}$ XVIII ${ }^{\mathrm{e}}$ siècle, un saut conceptuel permet d'affirmer que l'État est le lieu de transcendance, par sa capacité à dépasser

9. Eisenstadt et Meyer sont, l'un et l'autre, conscients de la modernité du concept de religion, c'est pourquoi ils le distinguent résolument de la forme (gestalt) prémoderne par exemple du christianisme. J. W. Meyer (1989: 401) souligne ainsi : "There is a tendency to think of Christendom and the Church as what we would now call a religious system, reaching back into history the more specialized notions we now attach to the term "religion" [...] Christendom, more reasonably, is seen through earlier (and probably modern) Western history as a much less differentiated, specialized, and organizationally structured system: more a polity than a "religion" (in the modern sense) "; voir également S. Eisenstadt, 1993.

10. A. Pizzorno $(1987: 33,44)$ souligne à juste titre que ce transfert de charisme du pouvoir " religieux " vers le pouvoir "séculier ", qui remonte aux réactions à la révolution grégorienne, n'est pas assimilable à une différenciation entre « religion » et « politique ». 
les oppositions sociales. Il s'agit bien là de privatisation (conceptuelle) de la « religion» (R. Koselleck, 1979).

En « dévoluant » l'autorité divine à l'État, la chrétienté a perdu de son importance à la fois en matière de légitimation du pouvoir politique et de constitution de regroupements. Cela n'implique cependant pas pour les références chrétiennes traditionnelles la perte de toute pertinence sociale. Au contraire, ce qui est désormais qualifié de "religion » peut être intégré à des projets de rationalisation et de discipline dans le cadre de l'État national. Ce dernier endosse alors un pouvoir de contrôle sur des domaines de la vie autrefois soumis au pouvoir " religieux ", comme l'éducation, le droit et la science - sans que leur contenu s'en trouve sensiblement changé dans un premier temps (H. Berman, 2002). Philip Gorski (2000 : 150 ; 2003) a même diagnostiqué, en examinant l'entrée dans la modernité des territoires luthériens, une "dédifférenciation » de la religion et de la politique ${ }^{11}$. Il montre comment on a fait appel à des symboles chrétiens pour construire des identités correspondant à des territoires nationaux, non seulement à l'époque confessionnelle où les princes déterminaient la "religion » de leurs sujets, mais aussi plus tard, dans ce que l'on appelle la « seconde époque confessionnelle » du XIX ${ }^{\mathrm{e}}$ siècle, au cours de laquelle nationalisation et confessionnalisation étaient étroitement imbriquées, comme le montre l'historiographie récente (O. Blaschke, 2000 ; P. van der Veer, H. Lehmann, 1999).

Sur cette toile de fond, il est évident que dans le discours auto-descriptif de la modernité, les définitions de la "religion» ont pris une importance centrale pour la constitution du politique et de ses limites. Les apports définitionnels scientifiques de l'histoire religieuse et de la sociologie des religions nées au $\mathrm{XIX}^{\mathrm{e}}$ siècle sont eux aussi une composante de ce discours. Ces disciplines n'ont pas simplement été le reflet des évolutions socioculturelles, mais elles ont aussi ouvert un horizon d'attente qui a orienté les pratiques sociales : c'est ce que montre en particulier le concept de "sécularisation " issu de la dichotomie sémantique entre "religion » et "séculier ». Dans une réinterprétation métaphorique d'un concept relevant d'abord du droit canon, puis lié au statut juridique des religions d'État, la «sécularisation "s'est transformée en un métarécit. Ce métarécit est en particulier reproduit par le discours sociologique de la modernité, avec des variantes nostalgiques et utopiques, marquées par la continuité et la discontinuité. Ce récit a légitimé des projets institutionnels de constitution moderne d'Étatsnations. Le transfert de charisme du pouvoir « religieux » vers le pouvoir " séculier » a en effet mené à des constructions culturelles du « séculier » qui ont donné

11. Quoique je partage la critique de Gorski à l'égard de la thèse de la différenciation comme centre paradigmatique de la théorie de la sécularisation, il me semble important de souligner que cette "différenciation " a elle aussi lieu dans un contexte modifié, à savoir dans le cadre de nouveaux imaginaires séculiers comme le souligne Charles Taylor (2007); voir aussi M. Koenig, 2012. 
une légitimité aux configurations institutionnelles d'un ordre politico-juridique et d'une identité collective dans l'État territorial et national, sans que cela n'implique nécessairement une différenciation entre «politique» et « religion ».

Des variantes institutionnelles de la sécularité sont visibles au sein même de la modernité européenne. En effet, du fait des structures de clivage confessionnel et du phénomène de path dépendance dans la constitution d'États-nations, divers arrangements institutionnels entre la politique et la religion se sont développés, comme la laïcité de la III ${ }^{\mathrm{e}}$ République française, les régimes juridiques de coopération entre État et Église, prédominants dans l'Europe centrale bi-confessionnelle, ou encore les Églises d'État [Staatskirche] ou Églises nationales [Volkskirche] scandinaves - arrangements qui témoignent d'une « remarquable imprégnation culturelle ", c'est-à-dire du poids des héritages religieux, comme le remarque Danièle Hervieu-Léger (2003 : 294). Depuis Tocqueville, on a beaucoup commenté la variante nord-américaine de la sécularité, qui combine une séparation stricte entre État et Églises et un faible niveau de différenciation entre politique et religion (S. Eisenstadt, 2000 : 64). Cependant c'est surtout dans des contextes non chrétiens que l'on trouve des variantes particulièrement marquantes, contextes dans lesquels d'autres formulations de la tension axiale entre sphères transcendante et immanente ont conduit, d'une perspective de la longue durée, à des constructions spécifiques de la sécularité pour l'époque moderne. Il est exclu de retracer ici les constructions culturelles et les variantes institutionnelles de la sécularité. Divers processus actuels de transformation des sécularités explicables par l'interaction entre la société mondiale et des trajectoires "civilisationnelles » spécifiques peuvent être cependant évoqués.

L'essor de mouvements religieux ou relevant d'un nationalisme religieux, en réaction contre le modèle occidental d'État-nation séculier, donne ainsi lieu à certaines reconfigurations institutionnelles entre religion et politique (W. Spohn, 2003 ; M. Juergensmeyer, 1993). Les changements des modèles légitimes de l'État-nation, observables principalement dans la période d'après-guerre, résultent pour l'essentiel de l'institutionnalisation mondiale des droits de l'Homme, qui a permis de renforcer le droit individuel à la liberté de religion, mais aussi des droits collectifs de minorités religieuses (et autres). Comme le constate de diverses manières la littérature consacrée au changement de la citoyenneté nationale (Y. Soysal, 1994 ; M. Koenig, 2007a), cette institutionnalisation mondiale des droits de l'Homme a conduit à dissocier les systèmes juridico-politiques et les identités collectives, et à intégrer les identités religieuses à la sphère publique. On attend ainsi des États qu'ils respectent la « diversité » culturelle et religieuse et même qu'ils en fassent activement la promotion. Or, la manière dont chaque État modifie la gouvernance de la diversité religieuse sous l'impact de ce discours mondial des droits de l'Homme s'avère nettement infléchie par sa trajectoire « civilisationnelle " spécifique. Ainsi, en Europe de l'Ouest, la redéfinition de l'État séculier dans un contexte de diversité religieuse intensifiée a donné lieu à 
la critique des configurations institutionnelles des relations entre État et Église(s) telles qu'elles s'étaient historiquement développées (voir M. Koenig, 2007b). Le débat français sur une "laïcité ouverte », le débat allemand sur la transformation du statut juridique de l'Église en un « droit constitutionnel religieux » pluraliste et le débat britannique sur un « disestablishment » de la religion anglicane, induits en particulier par la présence de l'islam résultant de l'émigration, indiquent la pression à laquelle sont soumises les configurations classiques. Les élites politiques et intellectuelles continuent toutefois de considérer la pluralité religieuse visible dans la sphère publique, sur fond d'imaginaire européen du séculier, comme une menace pour les identités nationales. L'appropriation de modèles (culturels) mondiaux de la diversité religieuse s'accompagne donc de conflits aigus sur les frontières symboliques entre religion et politique (ou sphère publique).

L'évolution du modèle mondial de l'État-nation a eu d'autres effets dans l'est de l'Asie. Dans le contexte d'une modernité post-confucéenne, la tension entre transcendance et immanence n'avait dans un premier temps pas comporté de différenciation entre pouvoir "séculier » et pouvoir "religieux ». Comme le montre le cas de la Corée (M. Koenig, 2000), la réception des modèles modernes de la "religion " a d'abord eu pour conséquence une très large restructuration des traditions. Suivant l'exemple du protestantisme (américain), dont les missionnaires avaient trouvé un large écho dès la fin du XIX ${ }^{\mathrm{e}}$ siècle ${ }^{12}$, est apparu en coréen aussi un terme distinct pour "religion" (chongyo $\left.{ }^{13}\right)$. À la suite de cela, non seulement les traditions locales sont réinterprétées en tant que « religions ", mais le pays connaît même une vague de création de nouvelles religions. L'invention du confucianisme comme " religion » est sans doute l'exemple le plus connu de l'influence du modèle culturel de religion mondial. Dans les années 1990, l'Académie confucéenne de Séoul tentait encore de "religionaliser » (sic) la tradition confucianiste, c'est-à-dire d'en fixer les convictions religieuses, les rites et les règles d'appartenance. L'institutionnalisation de catégories modernes du séculier et du religieux dans l'État-nation a donc été productive pour les religions, comme le succès du protestantisme le montre, tout en entraînant potentiellement de nouveaux conflits sur les limites de la liberté de religion. Ces conflits sont toutefois restés largement latents en Corée du Sud, car on pouvait se rattacher directement aux modèles de diversité religieuse légitimés dans la société mondiale, sur fond d'idées séculaires sur la coexistence des " trois enseignements »

12. Du fait de son esprit anti-japonais à l'époque coloniale, au début du $\mathrm{XX}^{\mathrm{e}}$ siècle, le protestantisme eut aussi une fonction importante ultérieurement dans le processus de constitution de la nation, fonction à laquelle la théologie Minjung et le mouvement de démocratisation purent se raccrocher dans les années 1970, mais nous ne pouvons que l'évoquer à la marge.

13. Chongyo, est le terme générique utilisé pour parler de religion. La particule gyo signifie école, doctrine et chong évoque ce qui est orthodoxe, reconnu. Les termes se rapportant à une religion précise se terminent par gyo (pulgyo : bouddhisme : yugyo, confucianisme). Ce terme générique apparu sur le tard est en soi un signe de la pluralisation religieuse [NdE]. 
(confucianisme, taoïsme et bouddhisme), et du fait que ces modèles étaient compatibles avec les symboles d'identité nationale. C'est précisément dans les années 1990 que la « diversité religieuse » (chongyo dawon chuoi) est devenue un topos de l'autodéfinition nationale dans une mesure inconnue en Europe, et ce, surtout parce que la multi-religiosité ne peut, comme dans l'est de l'Asie, y être pensée comme un phénomène individuel.

Des contradictions au sein de la world polity prennent un tour particulièrement manifeste dans les processus de transformation d'une modernité postislamique en Asie centrale (C. Hann, M. Pelkmans, 2009). Sur la toile de fond d'une islamisation tardive de la région au XIX siècle, inaugurée par la Russie tsariste, et d'une politique jacobine et totalitaire de sécularisation au sein de l'Union soviétique, la garantie du droit à la liberté de religion est devenue, dans le processus de constitution post-communiste des États et nations, un titre de légitimation important. Cela vaut par exemple pour l'élite politique de la République kirghize, qui depuis l'effondrement de l'Union soviétique s'est largement appuyée sur des modèles occidentaux de démocratie ${ }^{14}$. Une pression normative et des incitations politiques et économiques émanant des Nations Unies, de l'OSCE et notamment des États-Unis, pays d'origine des missionnaires protestants fondamentalistes extrêmement actifs dans la région dans les années 1990, ont conduit à ancrer dans les institutions des droits relatifs à la liberté religieuse. Ces derniers ont cependant fait du pays un refuge pour les mouvements islamistes persécutés sous le régime répressif de l'Ouzbékistan voisin, et cette évolution a débouché finalement sur une politique publique plus restrictive vis-à-vis des religions. La reprise formelle du discours mondial sur la « diversité religieuse » par les élites politiques et intellectuelles, tel qu'il apparaît dans les conférences régionales les plus diverses sur le « dialogue des civilisations » en Asie centrale, est donc de plus en plus dissociée des conflits locaux.

La théorie sociologique est actuellement confrontée à des défis de grande portée, provoqués par des expériences de décentrement de la modernité occidentale (S. Kaviraj, 2005 : 535). Ces nouvelles situations requièrent en effet des révisions de l'appareil conceptuel sociologique, lequel semble nous éloigner des problématiques propres à Weber, tout en restant fidèle au sens de sa formulation de l' « éternelle jeunesse » des sciences sociales et de la culture. Le projet weberien de macrosociologie historique comparative n'a cependant rien perdu de son attrait. On a tenté ici de montrer qu'une actualisation de ce projet peut s'appuyer sur la synthèse de deux éminents prolongements de la théorie weberienne de la rationalisation, qui, par différence avec des théories évolutionnistes en sociologie

14. Pour une analyse des conflits linguistiques dans le processus de constitution postsoviétique des États et nations, cf. M. Koenig, 1999. 
(N. Luhmann), sont particulièrement attentifs aux cadrages culturels et à la contingence historique de la modernité : la théorie néo-institutionnaliste de la société mondiale, de John W. Meyer, et l'analyse de la théorie culturelle des modernités multiples de Shmuel Eisenstadt. Nous avons montré comment la synthèse entre leurs deux approches était possible du fait de leurs prémisses communes, et comment elle était même nécessaire du fait de leurs lacunes complémentaires. Cette synthèse peut alors s'inscrire dans un programme de recherche macrosociologique sur les variantes institutionnelles de la modernité dans la société mondiale. Nous n'avons pu certes qu'esquisser les grandes lignes d'un tel programme. Plusieurs questions demeurent, telles que celles de l'analyse empirique et historique des imaginaires du « séculier » et de la "religion » dans la société-monde, par exemple à travers des évolutions de langage du droit international ou du droit constitutionnel dans la longue durée. Il en va de même pour l'analyse des mécanismes sociaux de transformation des arrangements institutionnels. Celle-ci requiert l'analyse de la configuration des intérêts des acteurs religieux et politique (A. Gill, 2008), ainsi que celle de leurs relations structurelles dans les champs juridiques et judiciaires (M. Koenig, à paraître). Enfin, c'est aussi le cas des changements de religiosité individuelle et collective qui restent au centre des débats en sociologie de la religion (D. Pollack, 2007).

Matthias KOENIG

Université de Göttingen

Institut de recherche Max Planck sur les sociétés multireligieuses et multiethniques, Allemagne mkoenig@uni-goettingen.de

\section{Bibliographie}

AlEXANDER Jeffrey C., 1992, "The Fragility of Progress: An Interpretation of the Turn Toward Meaning in Eisenstadt's Later Work », Acta Sociologica, 35, p. 85-94.

Asad Talal, 1993, Genealogies of Religion. Discipline and Reasons of Power in Christianity and Islam, Baltimore \& London, Johns Hopkins Univ. Press.

-, 2003, Formations of the Secular. Christianity, Islam, Modernity, Stanford, Stanford Univ. Press.

BeCKFord James A., 2003, Social Theory \& Religion, Cambridge, Cambridge Univ. Press. Berman Harold, 2002, Droit et révolution: l'impact des Réformes protestantes sur la tradition juridique occidentale, Librairie de l'univ. d'Aix-en-Provence.

Blaschke Olaf, 2000, Das 19. Jahrhundert: Ein Zweites Konfessionelles Zeitalter? Geschichte und Gesellschaft, 26, p. 38-75.

BuRKe Roland, 2010, Decolonization and the Evolution of International Human Rights, Philadelphia, Univ. of Pennsylvania Press.

Casanova José, 1994, Public Religions in the Modern World, Chicago, Chicago Univ. Press.

Dobbelaere Karel, 1981, Secularization. A Multi-Dimensional Concept, London, Sage. 
EIsenstadt Samuel N., 1973, Tradition, Change, and Modernity, New York et al., John Wiley \& Sons.

-, 1978, Revolution and the Transformation of Societies: A Comparative Study of Civilizations, New York, Free Press.

-, 1982, "The Axial Age: The Emergence of Transcendental Visions and the Rise of the Clerics ", Archives européennes de sociologie, 23, p. 299-314.

-, 1993, "Religion and the Civilizational Dimensions of Politics ", in Arjomand, Said Amir (dir.), The Political Dimensions of Religion, New York, State Univ. of New York Press, p. 13-39.

-, 1995, Power, Trust and Meaning. Essays in Sociological Theory and Analysis, Chicago, Univ. of Chicago Press.

-, 1998, Die Antinomien der Moderne. Die jakobinischen Grundzüge der Moderne und des Fundamentalismus. Heterodoxien, Utopismus und Jakobinismus in der Konstitution fundamentalistischer Bewegungen, Frankfurt a. M., Suhrkamp.

-, 2000, Die Vielfalt der Moderne, Weilerswist, Velbrück Wissenschaft.

-, 2003, Comparative Civilizations and Multiple Modernities, 2 vol., Leiden \& Boston, Brill.

Eisenstadt Shmuel N., Curelaru M., 1976, The Form of Sociology - Paradigms and Crises, New York, John Wiley \& Sons.

GiLl Anthony, 2008, The Political Origins of Religious Liberty, Cambridge, Cambridge Univ. Press.

GoRski Philip S., 2000, « Historicizing the Secularization Debate ", American Sociological Review, 65, p. 38-167.

-, 2003, The Disciplinary Revolution. Calvinism and the Rise of the State in Early Modern Europe, Chicago, Univ. of Chicago Press.

Hann Chris, Pelkmans Mathijs, 2009, «Realigning Religion and Power in Central Asia: Islam, Nation-state and (Post)Socialism », Europe-Asia Studies, 61/9, p. 1517-1541.

HeInTz Bettina, Müller Dagmar, Schiener Heike, 2006, « Menschenrechte im Kontext der Weltgesellschaft. Die weltgesellschaftliche Institutionalisierung von Frauenrechten und ihre Umsetzung in Deutschland, der Schweiz und Marokko ", Zeitschrift für Soziologie, 35, p. 424-447.

HeInTZ Bettina, MüNCH Richard, Tyrell Hartmann (dir.), 2005, Sonderheft Weltgesellschaft. Zeitschrift für Soziologie, Stuttgart, Lucius \& Lucius.

Hervieu-Léger Danièle, 1993, La Religion pour Mémoire, Paris, Les Éditions du Cerf.

-, 2003, "Pour une sociologie des "modernités religieuses multiples" : une autre approche de la "religion invisible" des sociétés européennes ", Social Compass, 50, p. 287-295.

JePperson Ronald, Meyer John W., 2011, « Multiple levels of analysis and the limitations of methodological individualism », Sociological Theory, 29, p. 54-73.

Juergensmeyer Mark, 1993, The New Cold War? Religious Nationalism Confronts the Secular State, Berkeley, Univ. of California Press.

Kaviraj Sudipta, 2005, "An Outline of a Revisionist Theory of Modernity ", Archives européennes de sociologie, 46, p. 497-526.

KNöBl Wolfgang, 2001, Spielräume der Modernisierung. Das Ende der Eindeutigkeit, Weilerswist, Velbrück Wissenschaft.

-, 2007, Die Kontingenz der Moderne. Wege in Europa, Asien und Amerika, Frankfurt a. M./New York, Campus. 
KoenIG Matthias, 1999, "Social Conditions for the Implementation of Linguistic Human Rights Through Multicultural Policies: The Case of the Kyrgyz Republic ", Current Issues in Language and Society, 6, p. 57-84.

-, 2000, «Religion and the Nation-State in South Korea: a Case of Changing Interpretations of Modernity in a Global Context ", Social Compass, 47, p. 551-570.

-, 2005, Eisenstadt Shmuel Noah, in Kaesler D. (dir.), Aktuelle Theorien der Soziologie, München, C.H. Beck, p. 41-63.

-, 2007a, "Institutionnalisation mondiale des droits de l'homme et constructions des identités collectives ", Droit et Société, 67, 2007, p. 673-694.

-, 2007b, «Europeanising the Governance of Religious Diversity? An Institutionalist Account of Muslim Struggles for Public Recognition ", Journal of Ethnic and Migration Studies, 33/6, p. 911-932.

-, 2012, "Jenseits der Säkularisierungstheorie? Zur Auseinandersetzung mit Charles Taylor ", Kölner Zeitschrift für Soziologie und Sozialpsychologie, 63/4, p. 649-673.

Koenig Matthias, Wolf Christof, 2013, "Religion und Gesellschaft - Aktuelle Perspektiven ", Kölner Zeitschrift für Soziologie und Sozialpsychologie, 65, 1, p. 1-23.

Koenig Matthias (à paraître), "Governance of Religious Diversity at the European Court of Human Rights ", in Kymlicka W., Bolden J. (dir.), International Approaches to Governing Ethnic Diversity, Oxford, Oxford Univ. Press.

KoselLeck Reinhard, 1979, Le règne de la critique, Paris, Éditions de Minuit.

Luckmann Thomas, 1991 [1967], Die unsichtbare Religion, Frankfurt a. M., Suhrkamp.

LuHMann Niklas, 1975, "Die Weltgesellschaft », in Soziologische Aufklärung 2. Aufsätze zur Theorie der Gesellschaft, Opladen, Westdeutscher Verlag, p. 51-71.

-, 1997, Die Gesellschaft der Gesellschaft, Frankfurt a. M., Suhrkamp.

MeYER John W., 1989, "Conceptions of Christendom: Notes on the Distinctiveness of the West ", in Kohn M. (dir.), Cross-National Research in Sociology, Newbury Park, Sage Publications, p. 395-413.

-, 1999, "The Changing Cultural Content of the Nation-State: A World Society Perspective ", in Steinmetz G. (dir.), State/Culture, State Formation after the Cultural Turn, Ithaca, Cornell Univ. Press, p. 123-143.

MeYer John W., Boli John, Thomas George M., 1987, « Ontology and Rationalization in the Western Cultural Account », in Thomas G. M., Meyer J. W., Boli J. (dir.), Institutional Structure. Constituting State, Society, and the Individual, Newbury Park, Sage Publications, p. 12-37.

Meyer John W., Boli John, Thomas George M., Ramirez Francisco O., 1997, «World Society and the Nation State ", American Journal of Sociology, 103, p. 144-181.

MeYer John W., Jepperson Ronald L., 2000, "The “Actors" of Modern Society: the Cultural Construction of Social Agency ", Sociological Theory, 18, p. 100-120.

MeYER John W., 2005, Weltkultur. Wie die westlichen Prinzipien die Welt durchdringen, Frankfurt a. M., Suhrkamp.

NASSEHI Armin, 2003, Offenheit und Geschlossenheit. Studien zur Theorie der modernen Gesellschaft, Frankfurt a. M., Suhrkamp.

Pizzorno Alessandro, 1987, "Politics Unbound ", in Maier C. S. (dir.), Changing Boundaries of the Political. Essays on the Evolving Balance Between the State and Society, Public and Private in Europe, Cambridge, Cambridge Univ. Press, p. 27-62. 
Pollack Detlef, 2003, Säkularisierung - ein moderner Mythos? Studien zum religiösen Wandel in Deutschland, Tübingen, Mohr Siebeck.

-, 2009, Rückkehr des Religiösen? Tübingen, Mohr Siebeck.

Schluchter Wolfgang, 1988, Religion und Lebensfübrung. Studien zu Max Webers Religions- und Herrschaftssoziologie (2 vol.), Frankfurt a. M., Suhrkamp.

SChmidt Volker H., 2006, "Multiple Modernities or Varities of Modernity? », Current Sociology, 54, p. 77-97.

SCHWINN Thomas, 2001, Differenzierung ohne Gesellschaft. Umstellung eines soziologischen Konzepts, Weilerswist, Velbrück.

-, 2003, "Kulturvergleich in der globalisierten Modern ", in Albert G., Bienfait A., Sigmund St., Wendt C. (dir.), Das Weber-Paradigma. Studien zur Weiterentwicklung von Max Webers Forschungsprogramm, Tübingen, Mohr Siebeck, p. 301-327.

-, 2005, "Weltgesellschaft, multiple Moderne und die Herausforderungen für die soziologische Theorie ", Zeitschrift für Soziologie, Sonderheft Weltgesellschaft, p. 205-222.

Sewell William H., 2005, Logics of History. Social Theory and Social Transformation, Chicago, Chicago Univ. Press.

Soysal Yasemin Nuhoglu, 1994, Limits of Citizenship. Migrants and Postnational Membership in Europe, Chicago, Chicago Univ. Press.

SPOHN Willfried, 2003, "Multiple Modernity, Nationalism and Religion: a Global Perspective ", Current Sociology, 51, p. 265-286.

STICHWEH Rudolf, 2000, Die Weltgesellschaft. Soziologische Analysen, Frankfurt a. M., Suhrkamp.

TaYlor Charles, 2007, A Secular Age, Cambridge, Mass., The Belknap Press of Harvard Univ. Press.

Thomas George, Meyer John W., 1984, "The Expansion of the State ", Annual Review of Sociology, 10, p. 461-482.

Tschannen Olivier, 1992, Les théories de la sécularisation, Genève, Droz.

van der Veer Peter, Lehmann Hartmut (dir.), 1999, Nation and Religion. Perspectives on Europe and Asia, Princeton, Princeton Univ. Press.

Wagner Peter, 2001, Theorizing Modernity: Inescapability and Attainability in Social Theory, London, Sage.

WitTRock Björn, 2000, "Modernity: One, None, or Many? European Origins and Modernity as a Global Condition ", Daedalus, 129, p. 31-60.

WoвbE Theresa, 2000, Weltgesellschaft, Bielefeld, Transcript Verlag.

Wohlrab-SAHr Monika, BurCHARdt Marian, 2011, «Vielfältige Säkularitäten. Vorschlag zu einer vergleichenden Analyse religiös-säkularer Grenzziehungen », Denkströme, 7, p. 9-27. 


\section{Modernités multiples et mondialisation}

Les récentes tentatives théoriques de reconstruire les explications weberiennes de la modernité ont apparemment pris deux directions opposées, soulignant les dynamiques globales de la rationalisation et l'émergence de multiples modernités. Cet article suggère que l'intégration de ces deux courants macrosociologiques ouvre de fructueuses perspectives de recherche pour les sociologues des religions. Elle est non seulement conceptuellement faisable, mais également nécessaire pour surmonter leurs limitations analytiques respectives. Le potentiel analytique d'une telle synthèse est ensuite illustré en esquissant les grandes lignes d'une sociologie comparative de la sécularisation, qui s'attarde sur la diffusion globale et sur la "dépendance au chemin emprunté " régionale.

Mots-clés : modernités multiples, néo-institutionnalisme, mondialisation, rationalisation, comparatisme.

\section{Multiple Modernities and Globalization}

Recent theoretical attempts of reconstructing the Weberian account of modernity have taken two seemingly opposite directions, highlighting global dynamics of rationalization and the emergence of multiple modernities, respectively. This article suggests that integrating both macro-sociological currents opens up fruitful research perspectives for sociologists of religion. It is argued that integrating world society and multiple modernities approaches is not only conceptually feasible but also necessary to overcome their respective analytical limitations. The analytical potential of such a synthesis is then illustrated by sketching the broad contours of a comparative sociology of secularization that is attentive to global diffusion and regional pathdependence alike.

Key words: multiple modernities, neo-institutionnalism, globalization, rationalization, comparatism.

\section{Modernidades múltiples y mundialización}

Los recientes intentos teóricos de reconstruir la perspectiva weberiana de la modernidad tomaron dos direcciones aparentemente opuestas, que destacan dinámicas globales de racionalización y la emergencia de modernidades múltiples, respectivamente. Este artículo sugiere que integrando ambas corrientes macro-sociológicas se abren perspectivas fructíferas para los sociólogos de la religión. Se argumenta aquí que la integración de los abordajes de la sociedad mundial y de las modernidades múltiples es no solamente realizable sino también necesario para superar sus respectivas limitaciones analiticas. El potencial analítico de esta sintesis es trabajada aqui esbozando gruesamente los contornos de una sociología comparativa de la secularización atenta a la la difusión global de lo que parece ser un camino de dependencia interregional. Palabras clave: modernidades múltiples, neo-institucionalismo, mundialización, racionalización, comparativismo. 
